\title{
Prise en compte des paramètres contextuels en radioécologie post-accidentelle*
}

\author{
Ph. RENAUD, H. MAUBERT, J.-C. BERNIÉ**
}

(Manuscrit reçu le 15 janvier 1997, accepté le 11 mars 1997)

RÉSUME Les conséquences d'un rejet radioactif accidentel sur les productions agricoles et d'élevage dépendent dans une large mesure du contexte dans lequel se sont effectués les dépôts de radioactivité : conditions et occurrence du dépôt dans les calendriers agricoles et d'affouragement des animaux. Ces paramètres contextuels sont une source de variation des résultats des modèles souvent plus grande que les incertitudes liées aux facteurs de transfert eux-mêmes. Ainsi, la contamination du lait de vache évolue sur plusieurs décades dans les mois qui suivent le dépôt en fonction des pratiques zootechniques d'alimentation des animaux. De même, un plant de blé qui recevrait un dépôt d'éléments radioactifs en phase de maturation aura, à la récolte, des grains $10^{3}$ à $10^{4}$ plus contaminés que si le dépôt était intervenu avant la montaison. Pour élaborer un outil d'aide à la décision comme le logiciel ASTRAL, il a été nécessaire de réaliser une modélisation de la prise en compte de ces paramètres contextuels. De plus, afín d'assister l'expert dans ses évaluations, des bases de données par défaut relatives aux pratiques agricoles et d'élevage sont proposées pour la France métropolitaine. Toutefois dans le cas d'un accident réel, pour affiner les résultats, il serait important de rapidement mettre à jour ces paramètres contextuels, qui peuvent varier d'une année à l'autre.

ABSTRACT The consequences on agricultural or breeding products of a radiogctive accidental release are strongly linked to the context in which radioactive deposits occur : for example conditions of deposition in relation with agricultural and feeding schedules. These contextual parameters lead to a variation of results given by models greater than transfer factors uncertainties. For example, cow milk contamination stretches on several decades during the months following deposit as a function of feeding practices. In the same way, if a wheat plant receives the deposit during grain developpement, harvested grains will have a contamination level $10^{3}$ to $10^{4}$ times higher than if deposition occurs before the growing stage. To create a decision support system like the ASTRAL software, the need to realise a modelling of these contextual parameters has been encountered. Moreover, to help experts for evaluations, default informations relative to agricultural and breeding practices for France are given through databases. However, in case of a real accident, it would be important to re-initialize these contextual parameters which change from year to year, in order to obtain more precise assessments.

* Article paraissant en publication accélérée.

** Institut de protection et de sûreté nucléaire, Département de protection de l'environnement, Service d'études et de recherches sur les transferts dans l'environnement, 92265 Fontenay-aux-Roses cedex. 


\section{Introduction}

L'importance des transferts des radionucléides dans l'environnement et les éléments de la chaîne alimentaire à la suite d'un rejet accidentel unique dépend dans une très large mesure de paramètres contextuels. Ils s'agit notamment des dates de dépôt par rapport à certaines dates clés de la saison agricole pour les végétaux ou des calendriers d'affouragement pour les animaux, ainsi que des conditions de dépôt : temps sec ou humide, hauteur et intensité des précipitations...

Les évaluations de modèles radioécologiques dynamiques comme le modèle allemand ECOSYS (Müller et Pröhl, 1993) le modèle britannique Farmland (Brown and Simmonds, 1995) ou le modèle ASTRAL (Maubert et al. 1996) développé par l'IPSN, ne seront satisfaisantes que si l'on dispose de renseignements précis sur les conditions d'application des équations et des valeurs des paramètres proposées par le modèle. Pour élaborer un outil d'aide à la décision comme ASTRAL, il a été nécessaire de réaliser une modélisation de la prise en compte des paramètres contextuels qui déterminent le choix des transferts à considérer et leur intensité.

Ce document a pour objet de souligner l'importance de ces paramètres, notamment au regard des autres paramètres radioécologiques, et de montrer comment ils sont automatiquement pris en compte dans le module de calcul du logiciel ASTRAL.

\section{Transfert aux végétaux}

\subsection{Phénomènes de transfert}

Suite à un dépôt accidentel de radioactivité sur une culture, l'activité massique de la production végétale à sa récolte peut résulter :

- d'un transfert foliaire direct dâ à l'interception par les feuilles et tiges d'éléments radioactifs contenus dans l'air ou dans l'eau de pluie au moment où s'effectuent les dépôts. Cette interception sera d'autant plus efficace que la culture est plus développée et couvre plus le sol. La migration de certains de ces éléments vers la partie consommable de la plante est appelée «translocation». Ce phénomène est plus intense durant la maturation du végétal (formation des grains par exemple);

- d'un transfert racinaire, lorsque les radionucléides ont pénétré suffisamment dans le sol pour qu'ils puissent y être puisés par la plante. Le labour est le plus souvent le facteur d'incorporation et d'homogénéisation des contaminants dans l'horizon racinaire ;

- de la remise en suspension, par le vent ou lors de travaux agricoles, d'éléments déposés sur le sol, ou encore de l'éclaboussement des feuilles, lors des pluies, par des particules de sol contaminées. 
Tous ces phénomènes n'ont pas la même intensité. A dépôt égal, le transfert direct sur une céréale en période de formation du grain, par exemple, amènera des concentrations de radionucléides dans la partie consommable entre $10^{2}$ et $10^{4}$ fois plus importantes que le transfert indirect via le sol et les racines. Le premier cas correspond à un dépôt en juin, le second à un dépôt en octobre, avant labours et semis.

Ces phénomènes ne peuvent être déterminés que par la connaissance des cycles végétatifs en relation avec les calendriers agricoles, la date du dépôt, et les valeurs des paramètres de transfert proposés par le modèle radioécologique.

\subsection{Modélisation adoptée}

\subsubsection{Dates clés pour la détermination des transferts}

La figure 1 représente les principales phases du cycle agricole pour les plantes cultivées annuelles.

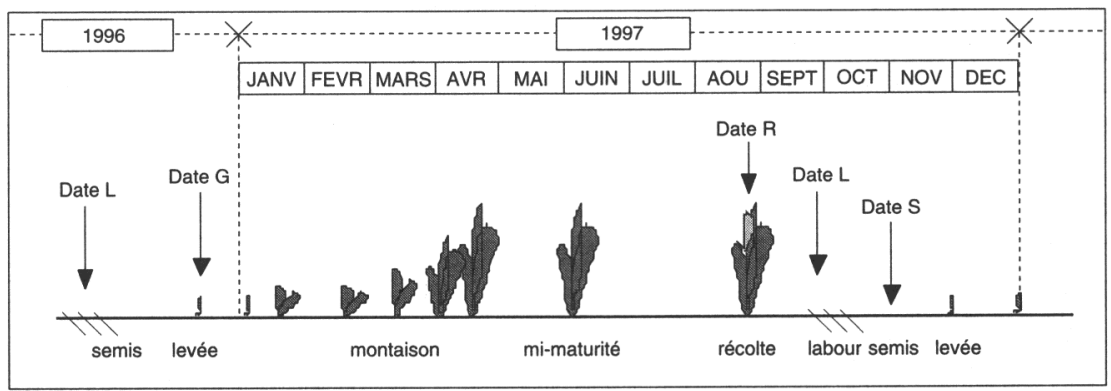

Fig. 1. - Cycle agricole pour les plantes annuelles. Agricultural cycle for annual plants.

Intéressons-nous à la récolte 97. Si le dépôt intervient avant le labour (date $L$ ), la plante sera contaminée par transfert racinaire. Par ailleurs, entre la levée (date $G$ ) et la récolte (date $R$ ) ses feuilles recevront des particules de sol contaminées remises en suspension ou projetées lors des pluies (éclaboussement). Ces transferts qui se font par l'intermédiaire du sol sont qualifiés de transferts indirects.

Si le dépôt a lieu après le labour et avant la levée, la contamination à la récolte résultera essentiellement de la remise en suspension des éléments radioactifs déposés à la surface du sol. Un dépôt postérieur à la levée entraîne une contamination par transfert foliaire direct. La contribution de ce transfert à la contamination de la plante à la récolte sera d'autant plus forte que le dépôt sera proche de celle-ci. Dans les deux cas, on parlera de transferts directs. 


\subsubsection{Equations de transfert utilisées}

La simplicité d'expression des équations est importante pour un modèle susceptible d'être utilisé en situation de crise par des utilisateurs plus ou moins familiarisés avec la radioécologie. Le modèle ASTRAL utilise essentiellement des paramètres globaux qui sont eux-mêmes des résultats de modélisations plus complexes qu'ils intègrent. Un facteur de transfert global est ainsi le plus simplement représentatif d'un ensemble de processus de transfert et permet de réduire les équations à leur plus simple expression.

Cas du transfert direct : la concentration d'une récolte ayant fait l'objet d'un transfert direct est calculée par l'équation (1).

où

$$
C_{\text {dir }}(t)=D \cdot \mathrm{FT}_{\mathrm{d}} \cdot \mathrm{e}^{-\lambda_{\mathrm{T}} \cdot t_{1}}
$$

$C_{\text {dir }}(t)$ : concentration due au transfert foliaire direct $\left(\mathrm{Bq} \mathrm{kg}^{-1}\right.$ frais)

$\mathrm{FT}_{\mathrm{d}}$ : facteur de transfert direct $\left(\mathrm{m}^{2} \mathrm{~kg}^{-1}\right)$

$\lambda_{\mathrm{r}}: \quad$ constante de décroissance radioactive $\left(\mathrm{j}^{-1}\right)$

$t_{1}$ : délai dépôt-récolte (j)

Le facteur de transfert global direct, $\mathrm{FT}_{\mathrm{d}}$, couvre à la fois les phénomènes d'interception, de translocation et de remise en suspension. Le modèle fournit ses valeurs tabulées pour différents délais avant récolte de manière à tenir compte du stade végétatif au moment du dépôt qui influe sur l'interception des radionucléides et sur leur translocation vers les parties comestibles (tableaux I et II). La détermination de sa valeur au moment du dépôt se fait automatiquement dans le module de calcul d'ASTRAL par interpolation linéaire entre les valeurs tabulées proposées par le modèle.

La remise en suspension est particulièrement significative lorsque le dépôt a lieu plus de 90 jours avant la récolte en raison de la faible contribution du transfert foliaire direct. Elle a été estimée en considérant que chaque jour a lieu un dépôt secondaire qui résulte de la redéposition des particules radioactives remises en suspension dans l'air. C'est donc $R_{\text {sus1, le résultat de l'équation }}$ (2), qui a été intégré au facteur de transfert global :

$$
\begin{gathered}
R_{\text {sus } 1}=\mathrm{e}^{-\lambda_{\mathrm{r}} \cdot t} \int_{0}^{t_{2}} T_{\text {rsus } 1} \cdot V_{\mathrm{rd}} \cdot R_{\mathrm{c}}(t) \cdot \mathrm{fte}(t) \cdot \mathrm{d} t \\
t_{2}=\inf \{\text { date } R \text {-date } D ; \text { date } R \text { - date } G \text { \} }
\end{gathered}
$$

avec

$\lambda_{\mathrm{r}}: \quad$ constante de décroissance radioactive $\left(\mathrm{j}^{-1}\right)$

$\mathrm{T}_{\text {rsus1 }}$ : coefficient de remise en suspension $\left(\mathrm{m}^{-1}\right)$

$V_{\mathrm{rd}}: \quad$ taux de redéposition $\left(\mathrm{m} \mathrm{s}^{-1}\right)$

$R_{\mathrm{c}}$ : $\quad$ rapport de captation

fte : facteur de translocation $\left(\mathrm{m}^{2} \mathrm{~kg}^{-1}\right)$

$t_{2}$ : $\quad$ durée de redéposition (j)

$R_{\mathrm{c}}$ et fte sont tabulés en fonction du délai avant récolte ; ce qui rend compte de l'évolution de l'interception par les végétaux de ces dépôts secondaires et de la translocation vers les parties comestibles au cours du temps. 
Cas du transfert indirect : la concentration d'une récolte qui a fait l'objet d'un transfert indirect est calculée par l'équation (3).

$$
\begin{aligned}
C_{\text {ind }}(t) & =D \cdot D_{\text {veg }} \cdot \mathrm{FT}_{\mathrm{i}} \cdot \mathrm{e}^{-\varphi \cdot t_{4}} \\
t_{4} & =\text { date } E-\text { date } D
\end{aligned}
$$

avec

$C_{\text {ind }}$ : concentration due au transfert indirect $\left(\mathrm{Bq} \mathrm{kg}^{-1}\right.$ frais)

$\mathrm{FT}_{\mathrm{i}}$ : facteur de transfert indirect $\left(\mathrm{m}^{2} \mathrm{~kg}^{-1}\right)$

$D_{\text {veg }}$ : facteur de dévégétation

$\varphi$ : taux de décroissance de l'activité disponible dans le sol $\left(\mathrm{an}^{-1}\right)$

$t_{4}$ : délai dépôt-étude (an)

Comme pour le transfert direct, l'équation est basée sur l'utilisation d'un facteur de transfert global, $\mathrm{FT}_{\mathrm{i}}$. Celui-ci rend compte de l'incorporation des radionucléides dans le sol et du transfert racinaire du sol vers les plantes. La diminution de la disponibilité pour les plantes des radionucléides contenus dans l'horizon racinaire (par éluviation, fixation aux particules de sol...) est paramétrée par un taux de clarence $\varphi$.

Pour estimer la fraction du dépôt qui est réellement parvenue au sol, il faut évaluer la fraction qui a été retenue par les végétaux et exportée lors de la première récolte. L'évaluation de l'interception initiale par la végétation en place au moment du dépôt ne suffit pas. En effet, le lessivage des feuilles conduit à une redistribution vers le sol. De plus une partie des résidus végétaux reste souvent sur le sol pour y être incorporée. Enfin, compte tenu de la rotation des cultures il est quasiment impossible de savoir quelle était la culture en place au moment du dépôt. Dans ASTRAL, on estime de manière arbitraire que si le dépôt a eu lieu durant la période estivale, pendant laquelle presque toutes les grandes cultures sont au maximum de leur développement foliaire, seule la moitié du dépôt est réellement parvenue au sol. C'est l'objet du paramètre contextuel $D_{\text {veg. }}$.

\subsubsection{Prise en compte des caractéristiques du dépôt}

L'interception par la masse foliaire des végétaux d'éléments radioactifs sous forme d'aérosols précipités par des gouttes de pluie ou en solution dans l'eau, est quelquefois moins efficace que celle d'aérosols qui se déposent par temps sec. Il en résulte que le transfert foliaire par voie humide peut être moins intense que le transfert par voie sèche. En effet les gouttes d'eau glissent sur les feuilles, ne laissant qu'un film actif dont l'épaisseur dépend du type de feuilles (configuration, inclinaison, état de surface), de la quantité d'eau tombée et de l'intensité de cette précipitation.

D'où la distinction de facteurs de transfert globaux propres aux dépôts par voie sèche et humide : respectivement $F_{\text {ds }}$ et $F T_{d h}$. Les valeurs de ces paramètres sont fournies en fonction du délai dépôt-récolte du radionucléide; mais aussi pour $\mathrm{FT}_{\mathrm{dh}}$, en fonction de la hauteur de précipitation (tableaux I et II). 


\section{TABLEAU I}

Valeurs du facteur de transfert global direct du césium par voie sèche : FT ds pour le blé d'hiver et la pomme de terre.

Values for the direct global transfer factor of cesium, dry conditions of deposition : FTds for winter wheat and potatoes.

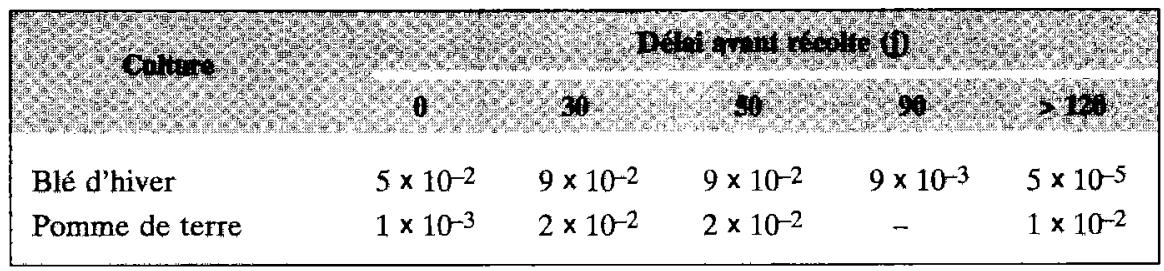

TABLEAU II

Valeurs du facteur de transfert global direct du césium par voie humide : FT dh pour 2 hauteurs de précipitation.

Values for the direct global transfer factor of cesium, wet conditions of deposition : FT dh for 2 amounts of rainfall.



Dans le modèle ASTRAL par exemple, on distingue quatre hauteurs de précipitation : $1 \mathrm{~mm}, 5,10$ et $15 \mathrm{~mm}$. La prise en compte de ce nouveau paramètre contextuel, déterminé par les conditions météorologiques lors du dépôt, se fait alors par une nouvelle interpolation sur la pluviométrie, des valeurs de $\mathrm{FT}_{\text {dh }}$ proposées.

Par ailleurs, il est peu fréquent qu'un dépôt s'effectue exclusivement par voie sèche ou par voie humide. La contribution de chacune des voies au dépôt total peut être estimée à partir des éléments météorologiques utilisés pour les calculs de transfert atmosphériques. Sa prise en compte dans le modèle de calcul d'ASTRAL se fait par la définition d'un paramètre, $K$, qui est la proportion du dépôt total qui s'est effectuée par voie sèche. Si le dépôt s'est fait uniquement par voie sèche, $K=1$. Si seulement $30 \%$ de l'activité s'est déposée par voie sèche, $K=0,3$. Le paramètre de transfert direct global, FT $_{d}$ défini précédemment se calcule alors par l'équation (4).

$$
\mathrm{FT}_{\mathrm{d}}=K \cdot \mathrm{FT}_{\mathrm{ds}}+(1-K) \cdot \mathrm{FT}_{\mathrm{dh}}
$$


FTds : Facteur de transfert global direct par voie sèche $\left(\mathrm{m}^{2} \mathrm{~kg}^{-1}\right)$

FT $_{\text {dh }}$ : Facteur de transfert global indirect par voie humide $\left(\mathrm{m}^{2} \mathrm{~kg}^{-1}\right)$

$K$ : $\quad$ proportion du dépôt total qui s'est effectuée par voie sèche

\subsubsection{Prise en compte des calendriers agricoles : exemples du blé d'hiver et des pommes de terre}

Afin de tenir compte des variations régionales, trois régions agro-climatiques ont été définies pour chaque production. Elles permettent de déterminer le calendrier agricole applicable en fonction de la région agro-climatique à laquelle appartiennent les zones touchées par les dépôts. La figure 2 présente à titre d'exemple le calendrier régional du blé d'hiver. Il apparaît que le calendrier agricole applicable à la région Rhône-Alpes est celui de la région agroclimatique 2 pour la partie nord, et 3 pour la partie sud.

Pour une même culture et dans une même région, les périodes de labour, de semis, de levée et de récolte s'étalent sur quelques semaines : deux ou trois le plus souvent. La figure 2 montre que la définition d'une date moyenne pour le labour, le semis ou la levée est suffisante compte-tenu des intensités relatives des transferts à ces périodes. En revanche la période de récolte peut s'étaler sur des périodes plus longues, plusieurs mois pour les pommes de terre par exemple, de fin août à fin novembre.

Le logiciel ASTRAL contient des données de ce type pour les principales productions agricoles françaises.

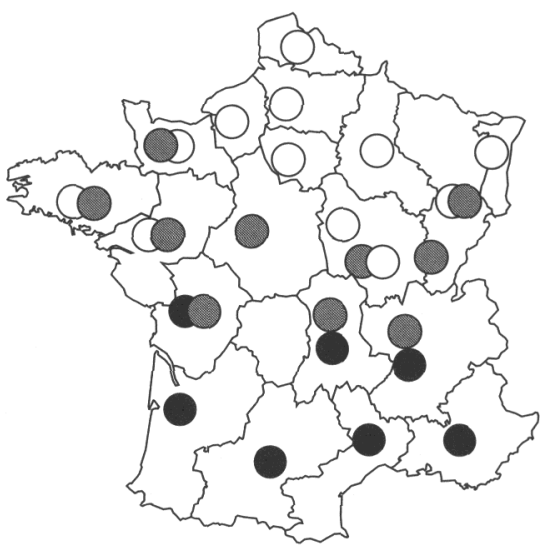

Blé d'hiver

\begin{tabular}{|cccc|}
\hline & labour & semis & récolte \\
\hline & 10.10 & 15.10 & $01.08-20.08$ \\
& 20.09 & 25.10 & $15.07-30.07$ \\
& 05.09 & 05.11 & $05.07-20.07$ \\
\hline
\end{tabular}

Fig. 2. - Calendriers agricoles régionaux du blé d'hiver (jours et mois). Agricultural regional schedules for winter wheat (day and month). 
Les deux exemples suivants montrent l'importance de ces calendriers.

La figure 3 représente l'évolution de la contribution des transferts à la concentration du ${ }^{137} \mathrm{Cs}$ dans les grains de blé à la récolte en fonction du délai dépôt-récolte. Le dépôt choisi est de $10^{6} \mathrm{~Bq} \mathrm{~m}^{-2}$.

Des aérosols déposés en septembre 96 (cas $\mathrm{D}_{1}$ ) seront incorporés dans l'horizon racinaire par le labour. La récolte 97 sera alors contaminée essentiellement par transfert racinaire avec une petite contribution de la remise en suspension de particules de sol. Dans ce cas la concentration attendue dans les grains sera de l'ordre de 30 à $90 \mathrm{~Bq} \mathrm{~kg}^{-1}$ suivant le type de sol.

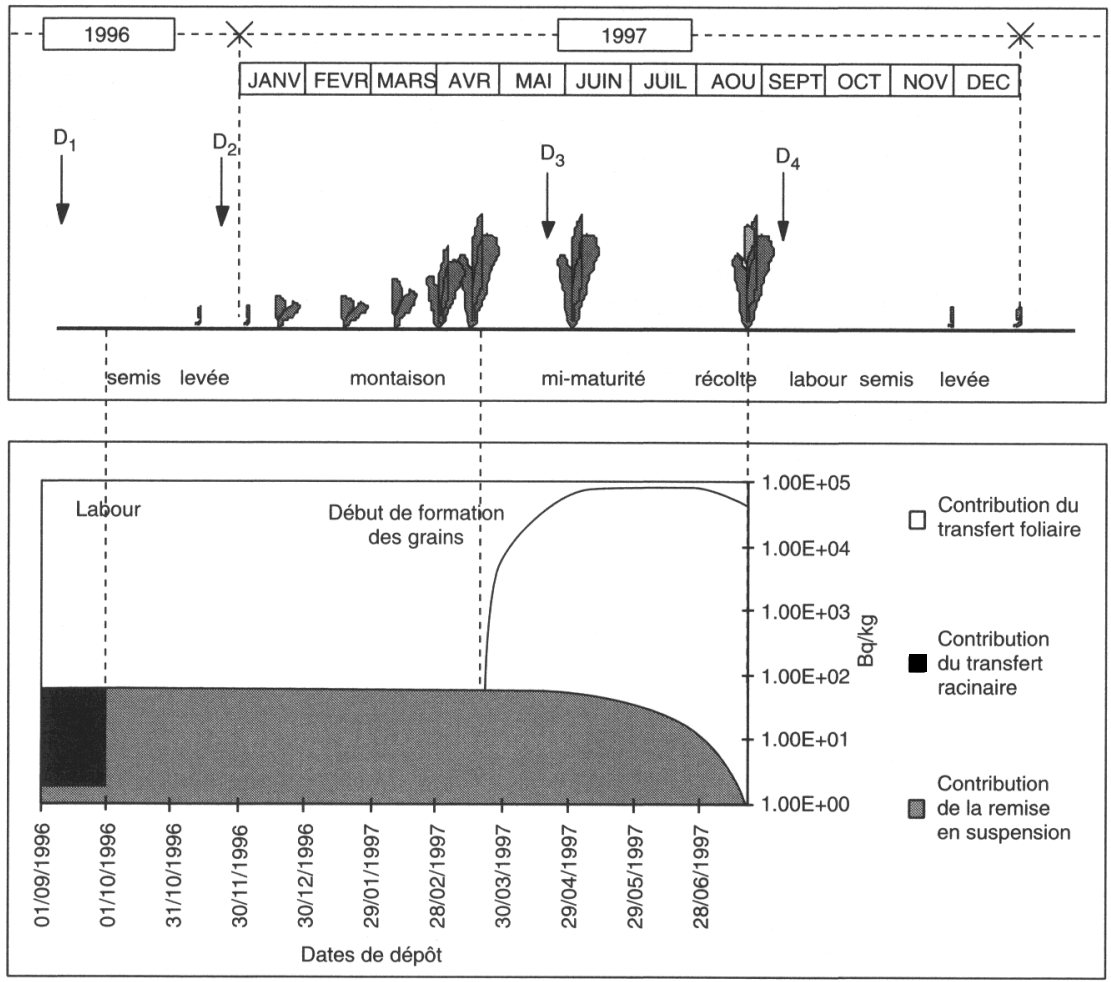

Fig. 3. - Contamination d'une récolte en fonction de la date d'occurrence du dépôt. Contamination of an harvest as a function of the date of the deposition. 
Si le dépôt intervient après le labour (cas $\mathrm{D}_{2}$ ), les aérosols resteront en surface. Même si la levée a déjà eu lieu, l'interception par les quelques feuilles (1 à 3 feuilles par plant à ce stade) sera très faible et l'essentiel du dépôt se fera au sol. Toutefois ce plant sera soumis dès la levée, à des phénomènes de dépôt, sur les feuilles, d'aérosols remis en suspension. Ces phénomènes augmentés d'un certain transfert par le sol, dit pseudo-racinaire car le labour n'a pas eu lieu, constituent dans ce cas les transferts prédominants qui contribuent pour l'essentiel à la contamination de la récolte qui sera du même ordre de grandeur que le transfert racinaire, soit $50 \mathrm{~Bq} \mathrm{~kg}^{-1}$ frais.

Si le dépôt se produit après la montaison, cas $D_{3}$, lorsque le plant a atteint son développement foliaire maximum, le transfert prédominant est le transfert foliaire direct résultant de l'interception des aérosols par les feuilles et tiges, puis de leur translocation vers le grain. Un dépôt dans les trois mois qui précèdent la récolte conduit à une contamination élevée des grains de 10000 à $100000 \mathrm{~Bq} \mathrm{~kg}^{-1}$ frais.

Si le blé est déjà récolté au moment du dépôt, il n'a fait l'objet d'aucun transfert et se trouve exempt de contamination.

La figure 4 présente l'évolution de la contamination des pommes de terre au cours de la période de récolte. Les pommes de terre les plus précoces sont celles qui présentent les niveaux de contamination les plus élevées (supérieures à $10^{4} \mathrm{~Bq} \mathrm{~kg}{ }^{-1}$ frais) car le dépôt est intervenu près de leur récolte. Celles récoltées fin novembre sont beaucoup plus faiblement contaminées $\left(20 \mathrm{~Bq} \mathrm{~kg}^{-1}\right.$ frais); elles n'étaient qu'au début de leur développement au moment du dépôt.

\section{Transfert aux produits d'origine animale}

\subsection{Modélisation dynamique des transferts aux produits animaux}

La modélisation des transferts aux produits animaux repose sur l'équation proposée dans le modèle ECOSYS (Müller et Pröhl, 1993).

$$
C_{\mathrm{a}}=\mathrm{FT}_{\mathrm{a}} \cdot \sum_{x=1}^{n} \int_{0}^{t_{4}} A_{\text {ing }}(t) \cdot \beta_{\mathrm{ax}} \cdot \lambda_{\mathrm{ax}} \cdot \mathrm{e}^{-\left(\lambda_{\mathrm{ax}}+\lambda_{\mathrm{r}}\right) \cdot\left(t_{3}-t\right)} \cdot \mathrm{d} t
$$

avec :

$C_{\mathrm{a}}(t)$ : concentration dans le produit animal $\left(\mathrm{Bq} \mathrm{l}^{-1}\right.$ ou $\left.\mathrm{Bq} \mathrm{kg}^{-1}\right)$

$\mathrm{FT}_{\mathrm{a}}$ : le facteur de transfert à l'équilibre $\left(\mathrm{j} \mathrm{kg}^{-1}\right)$

$A_{\text {ing }}$ : activité quotidiennement ingérée $\left(\mathrm{Bq} \mathrm{j}^{-1}\right)$

$\beta_{\mathrm{ax}}$ : coefficient métabolique

$\lambda_{\mathrm{ax}}$ : constante d'élimination de la fraction métabolisée $\mathrm{x}\left(\mathrm{j}^{-1}\right)$

$\lambda_{\mathrm{r}}$ : constante de décroissance radioactive $\left(\mathrm{j}^{-1}\right)$

$n$ : nombre de fractions métabolisées différentes $(n \leq 3)$

$t_{4}$ : délai dépôt-étude $(\mathrm{j})$ 


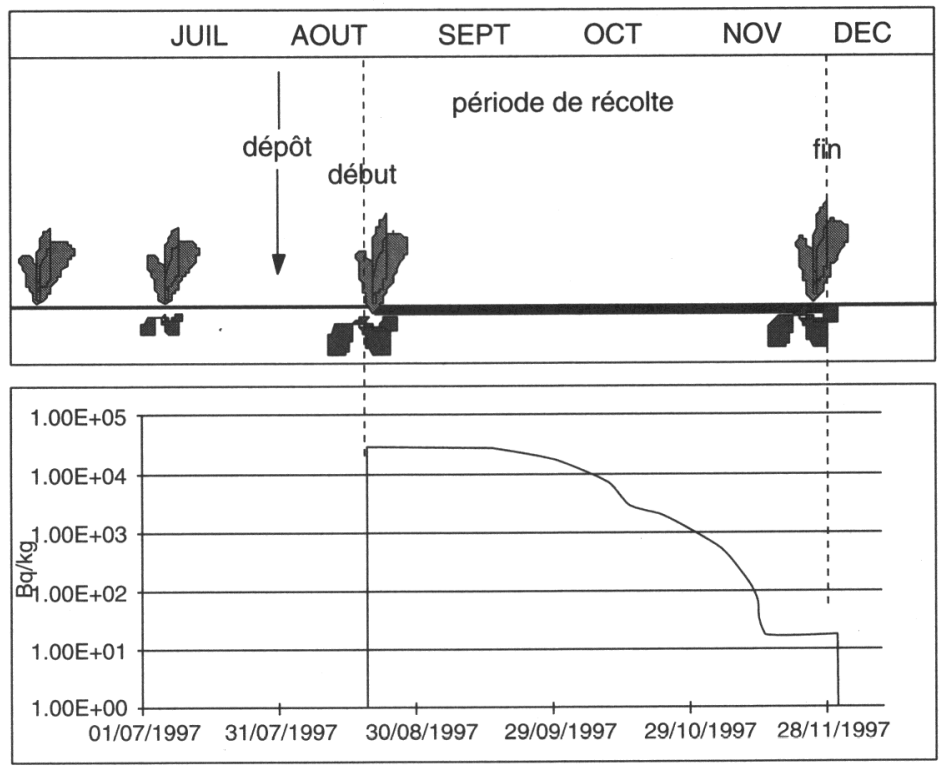

Fig. 4. - Contamination des pommes de terre récoltées tout au long de leur période de récolte.

Contamination of harvested potatoes all during their harvested period.

Elle se caractérise par une évaluation jour par jour de l'activité ingérée quotidiennement par l'animal : $A$ ing. Les autres termes sont relatifs à la métabolisation du radionucléide dans l'animal. Le facteur de transfert à l'équilibre $\mathrm{FT}_{\mathrm{a}}$, détermine la fraction de cette activité quotidiennement ingérée qui va parvenir au produit animal consommé ; par exemple le lait. Les termes intervenant dans les exponentielles rendent compte de la cinétique de ce transfert, c'est-à-dire de la vitesse avec laquelle le contaminant va circuler dans les autres organes et fluides avant de parvenir à l'organe cible. Pour l'exemple cité ici, les valeurs numériques des paramètres sont mentionnées dans le tableau III.

TABLEAU III

Valeurs des paramètres métaboliques pour le césium et le lait de vache. Values of metabolic parameters for cesium and cow milk.

\begin{tabular}{|lcccccc|}
\hline Radioélément & Produit & $\mathrm{FT}_{\mathrm{a}}$ & $\beta_{1}$ & $\lambda_{\mathrm{a} 1}\left(\mathrm{j}^{-1}\right)$ & $\beta_{2}$ & $\lambda_{\mathrm{a} 2}\left(\mathrm{j}^{-1}\right)$ \\
\hline Césium & Lait & $3 \times 10^{-3}$ & $8 \times 10^{-1}$ & $5 \times 10^{-1}$ & $2 \times 10^{-1}$ & $5 \times 10^{-2}$ \\
\hline
\end{tabular}


L'évaluation de la radioactivité quotidiennement ingérée, $A_{\text {ing, }}$ repose sur les calendriers d'affouragement qui définissent, au jour le jour, les quantités de fourrages consommées, $Q_{i}$.

$$
A_{\text {ing }}(t)=\sum_{i=1}^{4} Q_{i} . O_{i} \cdot C_{i}(t)
$$

avec :

$Q_{i}:$ quantité de l'aliment $i$ ingérée quotidiennement $(\mathrm{kg})$

$O_{i}$ : fraction de la ration alimentaire contaminée

$C_{i}$ : concentration de l'aliment $i$ au moment de sa consommation (Bq kg-1)

\subsection{Définition des calendriers d'affouragement dans ASTRAL}

Le module de calcul d'ASTRAL permet de définir jusqu'à 5 régimes d'affouragement par an, constitués chacun par un maximum de 5 fourrages choisis dans la liste suivante : maïs d'ensilage, maïs grain, orge d'hiver, escourgeon, oléagineux (tournesol, colza), protéagineux (pois et fèveroles), betteraves fourragères, herbe de pâture, foins de printemps et d'automne. La figure 5 présente un exemple de calendrier d'affouragement de vache laitière, tel qu'il apparaît dans le module de calcul d'ASTRAL. L'activité massique des fourrages au moment de leur consommation, $C(t)$, est fournie par les calculs de transferts aux végétaux avec prise en compte de la décroissance radioactive durant le délai récolte-consommation (sauf dans le cas de la pâture). Il est possible de tenir compte d'une fraction exogène non contaminée $O_{i}$ en introduisant l'activité massique correspondante. L'ingestion de particules de sol adhérentes aux aliments est également considérée; de même que la consommation d'eau contaminée. La concentration de l'eau n'est cependant pas calculée et est à introduire par l'utilisateur.

Des calendriers standards sont ainsi proposés à l'utilisateur qui peut les ajuster en fonction des informations dont il dispose (tableau IV). En effet les pratiques zootechniques sont complexes et sujettes à de plus grandes variations que les pratiques agricoles. Notamment pour les espèces bovines, les répartitions entre foins et ensilages en période hivernale peuvent être très différentes.

Le plus souvent, en absence d'informations précises, on doit se placer dans un contexte raisonnablement pessimiste. Il est recommandé par exemple de retenir suivant la date de l'accident, le foin susceptible d'être le plus contaminé : de printemps ou d'automne. La figure 6 présente l'évolution de la concentration en césium $137 \mathrm{du}$ lait de vache suite à un dépôt de $10^{4} \mathrm{~Bq} \mathrm{~m}^{-2}$ survenant un 15 juin. 


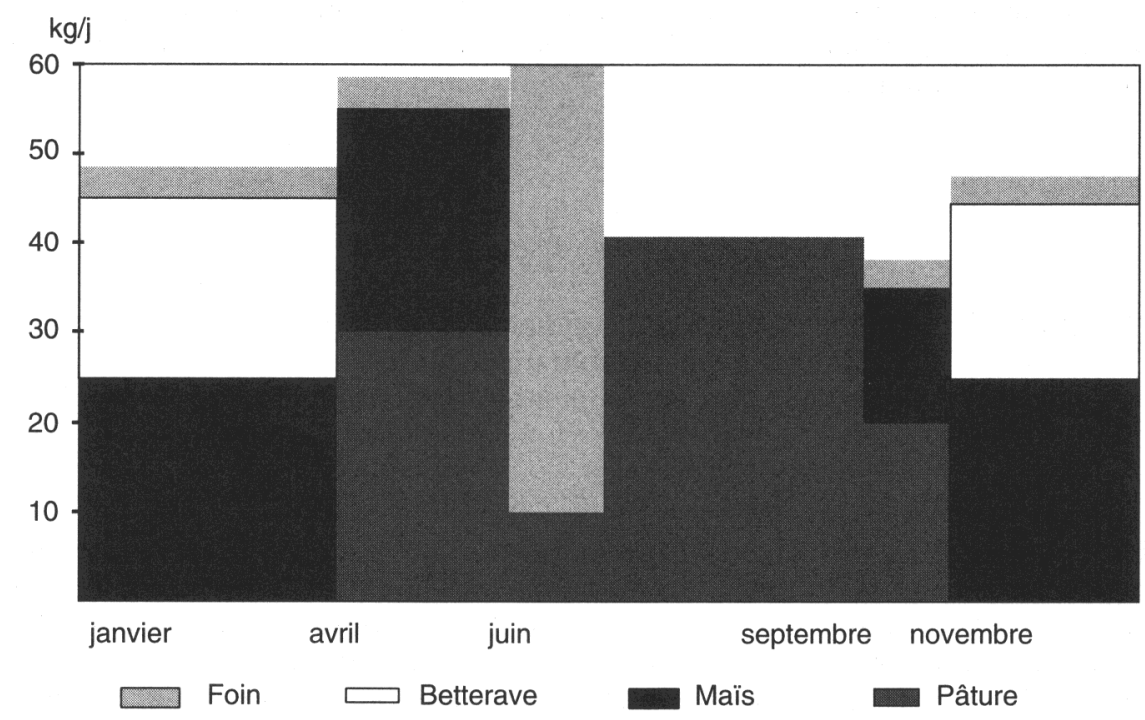

Fig. 5. - Calendrier d'affouragement de vaches laitières en Bourgogne. Feeding schedules for dairy cows in Bourgogne.

Après la période de pâturage durant laquelle la contamination du lait décroît avec celle de l'herbe, l'évolution en période hivernale est très différente (facteur 20 à partir de février) suivant que les foins utilisés pour l'affouragement des vaches aient été fanés fin juin ou fin septembre. La troisième courbe montre l'effet significatif d'un retrait du pâturage au bout de huit jours. L'application de contre-mesures conduit également à la définition de paramètres contextuels que le module de calcul d'ASTRAL prend automatiquement en compte (Renaud et al., in press).

Enfin il était important de montrer que l'incertitude sur un paramètre radioécologique aussi important que le facteur de transfert au lait, sujet très débattu dans les évaluations relatives aux rejets chroniques des installations nucléaires, ne présente dans le cas d'une évaluation post-accidentelle qu'un impact mineur au regard des paramètres contextuels que sont les pratiques zootechniques. C'est l'objet des barres d'incertitude définies pour l'une des courbes. Elles correspondent à un facteur d'incertitude de 2,25 (soit un écart d'approximativement un facteur 5 entre le minimum et le maximum) sur la valeur du facteur de transfert à l'équilibre. Bien que cette valeur ne résulte pas d'une étude statistique rigoureuse, elle constitue pour cette illustration, une évaluation acceptable de l'incertitude que l'on peut avoir sur ce paramètre radioécologique (Commisșion of the European Communities, 1991). 
TABLEAU IV

Exemples de calendriers d'affouragement $\left(\mathrm{kg} \mathrm{j}^{-1}\right)$. Examples of feeding schedules $\left(\mathrm{kg} \mathrm{j}^{-1}\right)$.

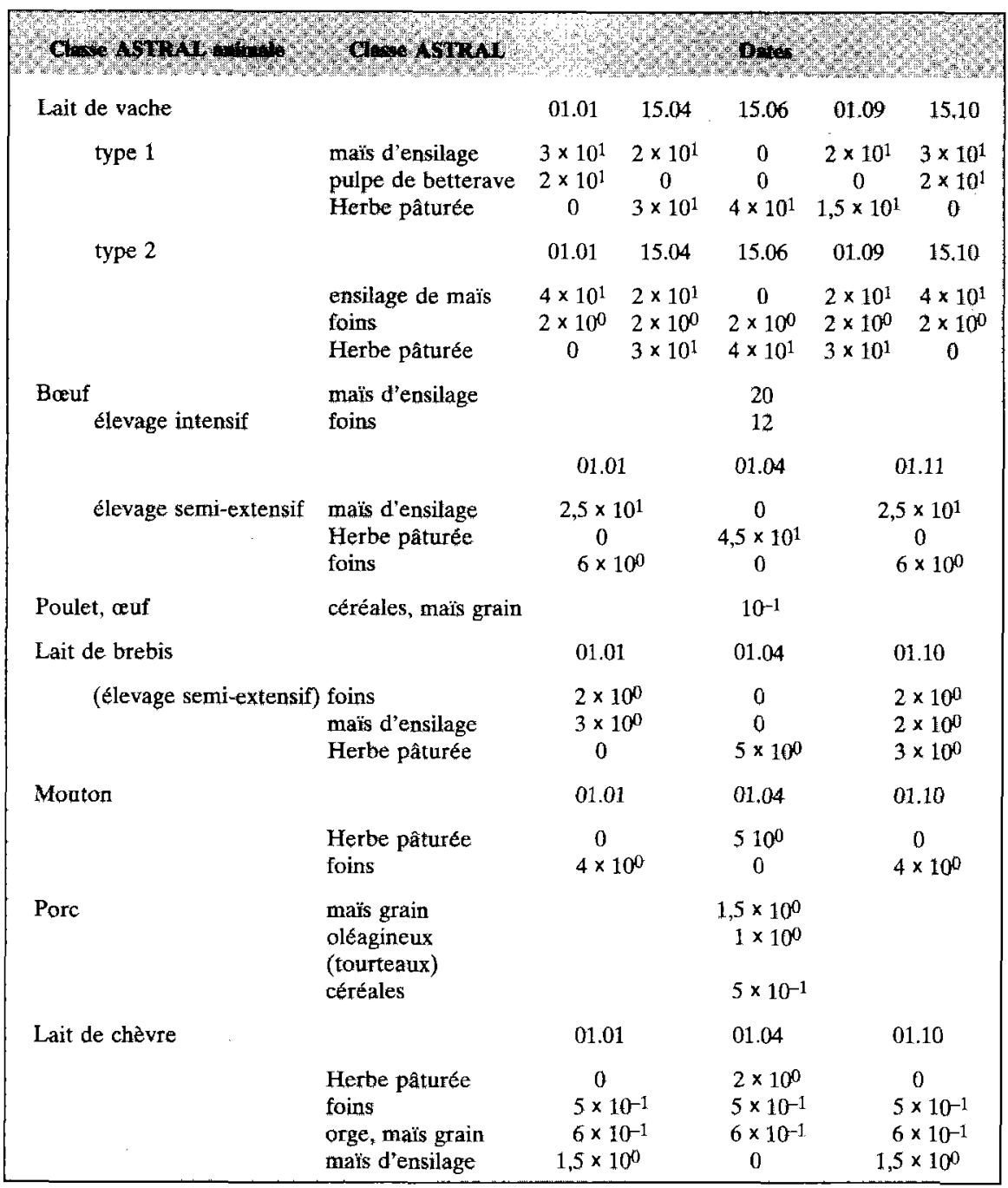




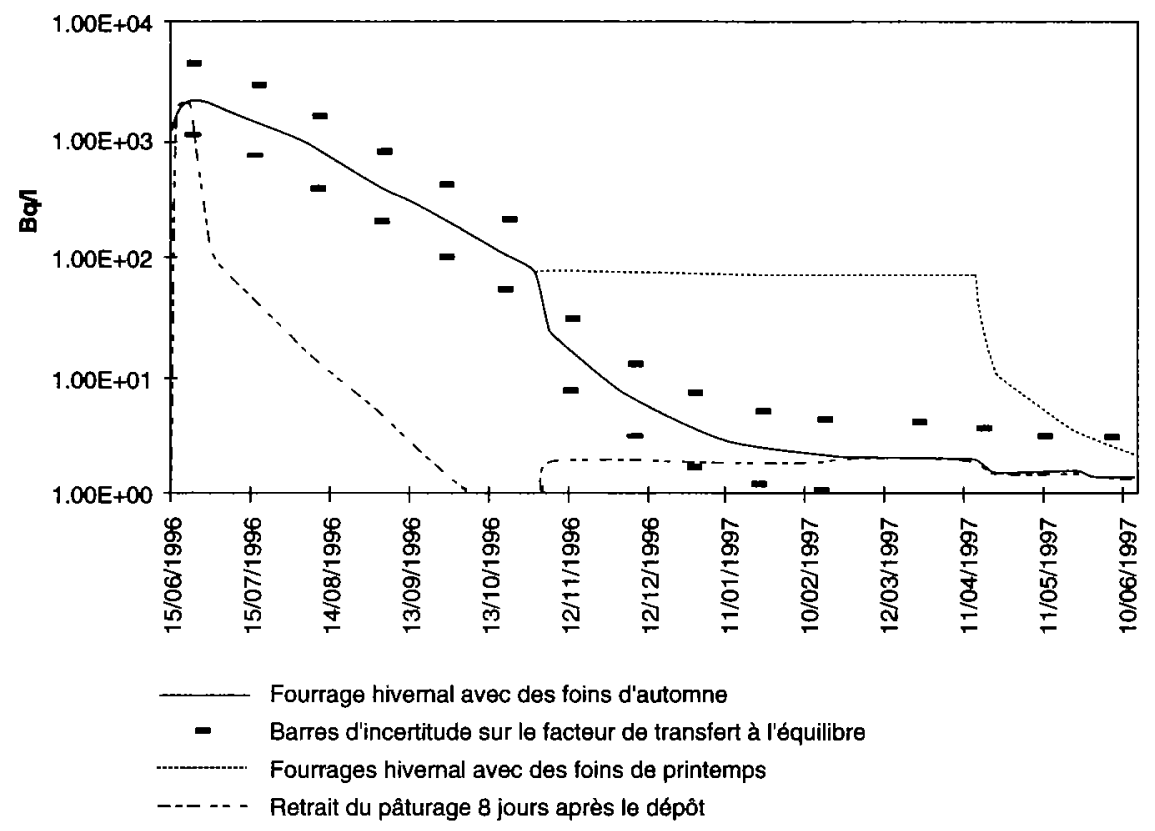

Fig. 6. - Evolution de la contamination du lait pour différents affouragements. Evolution of milk contamination for differents feeding schedules.

\section{Conclusion}

On a cherché ici à montrer que les modèles de transfert dynamiques des radionucléides dans l'environnement sont très sensibles aux paramètres contextuels, liés aux caractéristiques des dépôts et aux relations entre la date de dépôt, les calendriers agricoles et les pratiques zootechniques.

Ces paramètres sont une source de variation des résultats des modèles souvent plus grande que les incertitudes liées aux facteurs de transfert eux-mêmes, qui pour les radionucléides les plus importants en situation post-accidentelle, césium et strontium, sont relativement bien connus. Ainsi, la contamination du lait de vache évolue sur plusieurs décades dans les mois qui suivent le dépôt, avec de brusques inflexions résultant des pratiques zootechniques d'alimentation des animaux. De même, un plant de blé qui recevrait un dépôt d'éléments radioactifs en phase de maturation aura, à la récolte, des grains $10^{3}$ à $10^{4}$ plus contaminés que si le dépôt était intervenu avant la montaison. 
Dans le logiciel ASTRAL, une modélisation de la prise en compte de ces paramètres associée à des bases de données par défaut des pratiques agricoles et d'élevage a été réalisée afin d'assister l'expert dans ses évaluations. Toutefois dans le cas d'un accident réel, pour affiner les résultats, il serait important de rapidement mettre à jour ces paramètres contextuels, qui peuvent varier d'une année et d'une région à l'autre.

\section{RÉFÉRENCES}

Anonyme (1991) Radioactivity transfer to animal products. Radiation protection of the Commission of the European Communities. Rapport EUR 12608 EN.

Brown J., Simmonds J.R. (1995) FARMLAND, a dynamic model for the transfer of radionuclides through terrestrial foodchain, NRPB report $n^{\circ} \mathrm{R} 273$.

Maubert H., Renaud Ph., Fache Ph. (1996) ASTRAL : a software for the estimation of accidental releases of radionuclides in the environment. In : IRPA 9, Vienna, April 1996.

Múller H., Pröhl G. (1993) ECOSYS-87 : a dynamic Model for assessing radiological consequences of nuclear accidents. Health Phys. 64, (3), 232.

Renaud Ph., Maubert H. (1997) Agricultural counter-measures in the management of a post-accidental situation, J. Environ. Radioact, in press. 